\title{
Simultaneous exact model matching with stability by output feedback
}

\author{
Konstadinos H. Kiritsis *
}

\begin{abstract}
In this paper, is studied the problem of simultaneous exact model matching by dynamic output feedback for square and invertible linear time invariant systems. In particular, explicit necessary and sufficient conditions are established which guarantee the solvability of the problem with stability and a procedure is given for the computation of dynamic controller which solves the problem.
\end{abstract}

K e y w or d s: simultaneous model matching, output feedback, stability

\section{Introduction}

In this paper, we shall consider discrete-time, square and invertible, completely reachable and observable, linear time-invariant systems. The simultaneous exact model matching with stability consists in finding a single proper and stable output feedback controller that stabilizes a given finite set of square and invertible linear time-invariant systems and makes the closed-loop transfer function matrices equal to a given transfer function matrices. From the above it follows that the simultaneous exact model matching problem by dynamic output feedback contains the so-called simultaneous stabilization problem by dynamic output feedback.

The problem of simultaneous stabilization can be stated as follows: Does there exists a single controller which stabilizes a finite set of different linear timeinvariant systems? This is a very simple question in linear control theory but simple questions cannot always be simply answered [1]. The problem of simultaneous stabilization has a long history and is probably one of the most prominent open questions in linear control theory. A very short review of history of this longstanding open problem is given below.

The problem of simultaneous stabilization has been posed for the first time in [2]. The name, simultaneous stabilization has been introduced for the first time by authors of [3]. Necessary and sufficient conditions for the simultaneous stabilization of two systems by dynamic output feedback, have been established in [3] and [4]. In [5] is proved that the simultaneous stabilization of three systems is equivalent to partial pole placement by stable and minimum phase controller. An extension of the results of [5] to more than three plants has been given in [6]. In [7], has been proved that if a finite set of minimum phase systems, have the same number of transmission zeros, and the same high frequency sign, then there exists a single controller that stabilizes all given systems. In [8], has been showed that if a finite set of strictly proper and minimum phase, systems, have the same relative degree and the same high frequency sign, then the given systems can be simultaneously stabilized by stable and strictly proper controller.

In [9], has been derived a sufficient condition for the simultaneous stabilization of a finite number of single variable linear systems. In [10] and [11] iterative procedures are given for the computation of a strictly proper and stable controller that simultaneously stabilizes a finite set of strictly proper and minimum phase single variable and multivariable systems which have the same high frequency sign. In [6] has been proved that the problem of simultaneous stabilization of two systems by a stable controller is "rationally undecidable". This means that is required an infinite number of elementary operations, such as addition, substraction, multiplication, division, logical AND, and logical OR, to determine existence of the solution. From the above it follows that the solution of the simultaneous stabilization of three or more systems, that is equivalent to the strong simultaneous stabilization of two or more systems is also in general "rationally undecidable". Simultaneous stabilization is a hard [12], challenging and very important problem in linear control theory that has been extensively studied by many researchers but is still an open problem.

On the other hand, the exact model matching problem is a very broadly studied control problem. This is due to its great theoretical and practical importance. The exact model matching techniques have been applied to the design of many control problems [13]. Such problems are the decoupling problem, the model following problem, the model tracking problem, the servomechanisms problem, the partial model matching and the model reference adaptive control. A formulation and the solution of exact model matching problem by proportional state feedback

* Hellenic Air Force Academy Department of Aeronautical Sciences Division of Automatic Control Air Bose of Dekelia, 1010, Dekelia, Athens, Greece, kkyritsis.hafa@haf.gr 
problem have been given for the first time in [14]. It is pointed out that the model matching algorithm of [14] can be applied effectively to invertible systems only [15]. Later in [15] a complete solution to the exact model matching problem is given. Their method reduces the solution of the model matching problem to one of solving a set of linear algebraic equations. In [16] using the structure algorithm have been established necessary and sufficient conditions for the solution of exact model matching problem by state feedback and dynamic compensation.

In [17], necessary and sufficient conditions are established under which the action of a cascade compensator on a given system can be realized by a proportional state feedback. Using the results of [17], exact model matching has been studied in [18-20].

Furthermore, in [21] necessary and sufficient conditions have been established for the existence of solution of exact model matching with stability by dynamic output feedback. In [22] has been obtained a parametric solution of stable exact model matching problem using an algebraic approach. In [23-26] have been established necessary and sufficient conditions for the existence of stable solutions of exact model matching problem. For more complete references the reader is referred to the excellent survey paper [27].

In this paper, the problem of simultaneous exact model matching by dynamic output feedback for square and invertible, linear time - invariant systems, is studied and completely solved. In particular, explicit necessary and sufficient conditions are established which guarantee the solvability of simultaneous exact model matching problem with stability and a procedure is given for the computation of dynamic controller that solves the problem. Our method is based on coprime factorizations of proper rational matrices over the ring of proper and stable rational functions and is computationally simple.

\section{Problem statement}

Consider a finite set of $M(M \geq 2)$, discrete-time, square and invertible, completely reachable and observable, linear time-invariant systems described by the following state-space equations

$$
\begin{aligned}
\mathbf{x}_{i}(k+1) & =\mathbf{A}_{i} \mathbf{x}_{i}+\mathbf{B}_{i} u(k), \\
y(k) & =\mathbf{C}_{i} \mathbf{x}_{i}(k)+\mathbf{D}_{i} u(k)
\end{aligned}
$$

for $i=1,2, \ldots, M$, where $\mathbf{x}_{i}(k)$ are the state vectors of dimensions $n \times 1, u(k)$ is the vector of inputs of dimensions $m \times 1$ and $y(k)$ is the vector of outputs of dimensions $m \times 1$ and $\mathbf{A}_{i}, \mathbf{B}_{i}, \mathbf{C}_{i}$ and $\mathbf{D}_{i}$ are real matrices of dimensions $n \times n, \times m, m \times n$ and $m \times m$ respectively. The transfer functions matrices of systems described by state-space equations (1) and (2) are given by

$$
\mathbf{T}_{i}(z)=\mathbf{C}_{i}\left(\mathbf{I} z-\mathbf{A}_{i}\right)^{-1} \mathbf{B}_{i}+\mathbf{D}_{i}
$$

for $i=1,2, \ldots, M$. We also consider the control low

$$
\mathbf{u}(z)=-\mathbf{F}(z) \boldsymbol{y}(z)+\mathbf{v}(z)
$$

where $\mathbf{F}(z)$ is proper rational matrix of size $m \times m$ and $\mathbf{v}(z)$ is the reference input vector of size $m \times 1$. By applying the dynamic control low (4) to the systems (1) the transfer functions matrices of closed-loop systems are

$$
\mathbf{T}_{c i}(z)=\mathbf{T}_{i}(z)\left[\mathbf{I}+\mathbf{F}(z) \mathbf{T}_{i}(z)\right]^{-1}
$$

for $i=1,2, \ldots, M$. Let $\mathbf{M}_{i}(z)$ for $i=1,2, \ldots, M$, be proper and stable rational functions of dimensions $m \times m$.

The simultaneous exact model matching with stability by dynamic output feedback for square and invertible linear time-invariant systems can be stated as follows. Does there exists a dynamic output feedback (4) with proper and stable $\mathbf{F}(z)$ such that the closed-loop systems with transfer functions given by (5), are proper and stable and

$$
\mathbf{T}_{i}(z)\left[\mathbf{I}+\mathbf{F}(z) \mathbf{T}_{i}(z)\right]^{-1}=\mathbf{M}_{i}(z)
$$

$\forall i=12, \ldots, M$ ? If so, give necessary and sufficient conditions for existence and a procedure to find it.

\section{Basic concepts and preliminary results}

Let us first introduce some notions that are used frequently throughout the paper. Let $\mathbb{R}$ be the field of real numbers. Also let $\mathbf{R}_{\mathrm{ps}}(z)$, be the ring of proper and stable rational functions in $z$. Two proper and stable rational matrices $\mathbf{A}(z)$ and $\mathbf{B}(z)$ having the same numbers of columns are said to be relatively right prime over $\mathbf{R}_{\mathrm{ps}}(z)$ if and only if there are matrices $\mathbf{X}(z)$ and $\mathbf{Y}(z)$ over $\mathbf{R}_{\mathrm{ps}}(z)$ such that

$$
\mathbf{X}(z) \mathbf{A}(z)+\mathbf{Y}(z) \mathbf{B}(z)=\mathbf{I}
$$

where $\mathbf{I}$ is the identity matrix of dimensions $r \times r, r$ is the number of columns of $\mathbf{A}(z)$ and $\mathbf{B}(z)$.

Definition 1. The matrices $\mathbf{D}(z)$ and $\mathbf{N}(z)$ over $\mathbf{R}_{\mathrm{ps}}(z)$ of dimensions $m \times m$ and $p \times m$ respectively with nonsingular $\mathbf{D}(z)$ form a right coprime factorization over $\mathbf{R}_{\mathrm{ps}}(z)$ of the proper rational matrix $\mathbf{T}(z)$ of dimensions $p \times m$ if and only if

(a) The matrices $\mathbf{D}(z)$ and $\mathbf{N}(z)$ are relatively right prime over $\mathbf{R}_{\mathrm{ps}}(z)$

(b) $\mathbf{T}(z)=\mathbf{N}(z) \mathbf{D}^{-1}(z)$

Consider a discrete-time, completely reachable and observable linear time-invariant system described by the following state-space equations

$$
\begin{aligned}
\mathbf{x}(k+1) & =\mathbf{A} \boldsymbol{x}(k)+\mathbf{B} \mathbf{u}(k), \\
\boldsymbol{y}(k) & =\mathbf{C} \mathbf{x}(k)+\mathbf{D} \mathbf{u}(k),
\end{aligned}
$$


Let $\mathbf{F}$ be a real matrix such that $(\mathbf{A}+\mathbf{B F})$ is stable (all eigenvalues in $|z|<1$ ) and let $\mathbf{K}=\mathbf{C}+\mathbf{D F}$. Then the matrices [28]

$$
\begin{aligned}
& \mathbf{D}(z)=\mathbf{F}[\mathbf{I} z-\mathbf{A}-\mathbf{B F}]^{-1} \mathbf{B}+\mathbf{I} \\
& \mathbf{N}(z)=\mathbf{K}[\mathbf{I} z-\mathbf{A}-\mathbf{B F}]^{-1} \mathbf{B}+\mathbf{D}
\end{aligned}
$$

form a right coprime factorization over $\mathbf{R}_{\mathrm{ps}}$ of system (9).

The proper and stable rational matrix $\mathbf{U}(z)$ of dimensions $m \times m$ whose inverse exists and is also proper and stable is called biproper and bistable. The following Lemma is needed to prove the main theorem of this paper [29].

Lemma 1. Let $\mathbf{D}(z)$ and $\mathbf{N}(z)$ be a right coprime factorization over $\mathbf{R}_{\mathrm{ps}}$ of system (9). Then there exists a proper and stable controller (4) that stabilizes system (9) if and only if the matrix $[\mathbf{D}(z)+\mathbf{F}(z) \mathbf{N}(z)]$ is biproper and bistable.

\section{Main results}

The main result of the paper is given below and gives explicit necessary and sufficient conditions for solvability of simultaneous exact model matching with stability, by dynamic output feedback for square and invertible linear time-invariant systems.

Theorem 1. Let $\mathbf{D}_{i}(z), \mathbf{N}_{i}(z)$ for $i=1,2, \ldots, M$ be a right coprime factorizations over $\mathbf{R}_{\mathrm{ps}}(z)$ of square and invertible, completely reachable and observable systems (1). Also let $\mathbf{M}_{i}(z)$ for $i=1,2, \ldots, M$ be proper and stable rational functions of dimensions $m \times m$. Then the simultaneous exact model matching with stability, by dynamic output feedback has a solution if and only if

(a) The matrices $\left[\mathbf{M}_{i}^{-1}(z) \mathbf{N}_{i}(z)\right]$ are biproper and bistable $\forall i=1,2, \ldots, M$.

(b) The matrices $\left[\mathbf{M}_{i}^{-1}(z)-\mathbf{T}_{i}^{-1}(z)\right]$ are proper and stable $\forall i=1,2, \ldots, M$.

(c) $\left[\mathbf{M}_{1}^{-1}(z)-\mathbf{T}_{1}^{-1}(z)\right]=\left[\mathbf{M}_{i}^{-1}(z)-\mathbf{T}_{i}^{-1}(z)\right]$, $\forall i=1,2, \ldots, M$.

Proof. To establish necessity, suppose that the problem of simultaneous exact model matching with stability has a solution by dynamic output feedback (4) with $\mathbf{F}(z)$ to be proper and stable. Then from (6) we have that

$$
\mathbf{T}_{i}(z)\left[\mathbf{I}+\mathbf{F}(z) \mathbf{T}_{i}(z)\right]^{-1}=\mathbf{M}_{i}(z), \forall i=1,2, \ldots, M
$$

Let $\mathbf{D}_{i}(z), \mathbf{N}_{i}(z)$ for $i=1,2, \ldots, M$ be a right coprime factorization over $\mathbf{R}_{\mathrm{ps}}(z)$ of square and invertible, completely reachable and observable systems (1) with transfer functions $\mathbf{T}_{i}(z)$ given by $(3)$.

Then from relationship (8) we have that for $i=$ $1,2, \ldots, M$

$$
\mathbf{T}_{i}(z)=\mathbf{N}_{i}(z) \mathbf{D}_{i}^{-1}(z)
$$

Equation (13) using relationship (14) is rewritten as

$$
\mathbf{N}_{i}(z)\left[\mathbf{D}_{i}(z)+\mathbf{F}(z) \mathbf{N}_{i}(z)\right]^{-1}=\mathbf{M}_{i}(z) .
$$

Since by assumption the systems (14) are invertible, we have that the transfer function matrices $\mathbf{T}_{i}(z)$ and the matrices $\mathbf{N}_{i}(z)$ are also invertible $\forall i=1,2, \ldots, M$. and therefore from (15) we have

$$
\left[\mathbf{D}_{i}(z)+\mathbf{F}(z) \mathbf{N}_{i}(z)\right]^{-1}=\mathbf{N}_{i}^{-1}(z) \mathbf{M}_{i}(z)
$$

or equivalently

$$
\mathbf{D}_{i}(z)+\mathbf{F}(z) \mathbf{N}_{i}(z)=\mathbf{M}_{i}^{-1}(z) \mathbf{N}_{i}(z) \text {. }
$$

Since the closed-loop systems (5) are stable and the matrix $\mathbf{F}(z)$ is by assumption proper and stable, from Lemma 1 we have that the matrices $\left[\mathbf{D}_{i}(z)+\mathbf{F}(z) \mathbf{N}_{i}(z)\right]$ are biproper and bistable $\forall i=1,2, \ldots, M$. This implies that the matrices $\mathbf{M}_{i}^{-1}(z) \mathbf{N}_{i}(z)$ must be also biproper and bistable $\forall i=1,2, \ldots, M$. This is condition (a) of Theorem 1. Equation (13) can be rewritten as follows

$$
\left[\mathbf{I}+\mathbf{F}(z) \mathbf{T}_{i}(z)\right]^{-1}=\mathbf{T}_{i}^{-1}(z) \mathbf{M}_{i}(z)
$$

or equivalently

$$
\mathbf{F}(z) \mathbf{T}_{i}(z)=\mathbf{M}_{i}^{-1}(z) \mathbf{T}_{i}(z)-\mathbf{I}
$$

or equivalently

$$
\mathbf{F}(z)=\mathbf{M}_{i}^{-1}(z)-\mathbf{T}_{i}^{-1}(z) .
$$

Since the matrix $\mathbf{F}(z)$ is by assumption proper and stable the matrices $\mathbf{T}_{i}^{-1}(z)-\mathbf{M}_{i}^{-1}(z)$ must be also proper and stable $\forall i=1,2, \ldots, M$. This is condition (b) of the Theorem. From equations (20) we have that

$$
\mathbf{F}(z)=\mathbf{M}_{i}^{-1}(z)-\mathbf{T}_{i}^{-1}(z) \forall i=1,2, \ldots, M
$$

and

$$
\mathbf{F}(z)=\mathbf{M}_{1}^{-1}(z)-\mathbf{T}_{1}^{-1}(z) .
$$

The relationships (21) and (22) have the same left sides and therefore

$$
\mathbf{M}_{1}^{-1}(z)-\mathbf{T}_{1}^{-1}(z)=\mathbf{M}_{i}^{-1}(z)-\mathbf{T}_{i}^{-1}(z) \forall i=2, \ldots, M
$$

This is condition (c) of the Theorem. The sufficiency of conditions (a), (b) and (c) of Theorem 1 can be proved as follows. Let

$$
\mathbf{F}(z)=\mathbf{M}_{1}^{-1}(z)-\mathbf{T}_{1}^{-1}(z) .
$$

Condition (b) of Theorem 1 guarantees that the matrix $\mathbf{F}(z)$ given by (24) is proper and stable. Using (14) we have

$$
\begin{array}{r}
\mathbf{D}_{1}(z)+\mathbf{F}(z) \mathbf{N}_{1}(z)=\mathbf{D}_{1}(z)+\left[\mathbf{M}_{1}^{-1}(z)-\mathbf{T}_{1}^{-1}(z)\right] \mathbf{N}_{1}(z) \\
=\mathbf{M}_{1}^{-1}(z) \mathbf{N}_{1}(z),
\end{array}
$$




$$
\begin{aligned}
& \mathbf{T}_{1}(z)\left[\mathbf{I}+\mathbf{F}(z) \mathbf{T}_{1}(z)\right]^{-1}= \\
& \quad \mathbf{T}_{1}(z)\left[\mathbf{I}+\left[\mathbf{M}_{1}^{-1}(z)-\mathbf{T}_{1}^{-1}(z)\right] \mathbf{T}_{1}(z)\right]^{-1}=\mathbf{M}_{1}(z) .
\end{aligned}
$$

Furthermore, from condition (c) of Theorem 1 and relationship (14) we have

$$
\begin{aligned}
& \mathbf{D}_{i}(z)+\mathbf{F}(z) \mathbf{N}_{i}(z)=\mathbf{D}_{i}(z)+\left[\mathbf{M}_{1}^{-1}(z)-\mathbf{T}_{1}^{-1}(z)\right] \mathbf{N}_{i}(z)= \\
& \mathbf{D}_{i}(z)+\left[\mathbf{M}_{i}^{-1}(z)-\mathbf{T}_{i}^{-1}(z)\right] \mathbf{N}_{i}(z)=\mathbf{M}_{i}^{-1}(z) \mathbf{N}_{i}^{-1}(z),(27)
\end{aligned}
$$

$$
\begin{array}{r}
\mathbf{T}_{i}(z)\left[\mathbf{I}+\mathbf{F}(z) \mathbf{T}_{i}(z)\right]^{-1}=\mathbf{T}_{i}(z)[\mathbf{I}+ \\
\left.\left[\mathbf{M}_{1}^{-1}(z)-\mathbf{T}_{1}^{-1}(z)\right] \mathbf{T}_{i}(z)\right]^{-1}=\mathbf{T}_{i}(z)[\mathbf{I}+ \\
\left.\left[\mathbf{M}_{i}^{-1}(z)-\mathbf{T}_{i}^{-1}(z)\right] \mathbf{T}_{i}(z)\right]^{-1}
\end{array}
$$

$\forall i=2, \ldots, M$. Since by condition (a) the matrices $\left.\mathbf{M}_{i}^{-1}(z) \mathbf{N}_{i}(z)\right]$ are biproper and bistable, $\forall i=1,2, \ldots, M$ from (25) and (27) and Lemma 1 we have that the dynamic controller (4) with proper and stable matrix $\mathbf{F}(z)$ given by (24), simultaneously stabilizes all closed-loop systems (5) and from (26) and (28) we have that the dynamic controller (4) with proper and stable matrix $\mathbf{F}(z)$ given by (24), satisfies all model matching equations (6). This completes the proof.

R e mark . As stated in introduction of this paper, the simultaneous exact model matching problem by dynamic output feedback contains the so-called simultaneous stabilization problem by dynamic output feedback. It is pointed out that the simultaneous stabilization problem by dynamic output feedback in its full generality is extremely hard problem [6] and [12] This means that it is extremely difficult to obtain an efficient algorithm for the solution of the problem in its full generality. From the above it follows that the simultaneous stabilization problem by dynamic output feedback can be studied using linear matrix inequality (LMI) approach, only for special classes of linear time-invariant systems.

\section{Computation of the controller}

In this section is described step by step the procedure for the computation of the proper and stable controller (4) that solves the simultaneous exact model matching problem with stability.

Given: $M(M \geq 2)$ linear square and invertible systems, described in state-space by the equations (1) and (2) and proper and stable rational matrices $\mathbf{M}_{i}(z)$ for $i=$ $1,2, \ldots, M$.

Find: A proper and stable matrix $\mathbf{F}(z)$ such that (6) hold. Step 1: Compute the transfer functions matrices of the given systems (1)

$$
\mathbf{T}_{i}(z)=\mathbf{C}_{i}(z)\left(\mathbf{I} z-\mathbf{A}_{i}\right)^{-1} \mathbf{B}_{i}+\mathbf{D}_{i} \text { for } i=1,2, \ldots, M .
$$

Step 2: Using (11) and (12) compute proper and stable rational matrices $\mathbf{D}_{i}(z), \mathbf{N}_{i}(z)$ for $i=1,2, \ldots, M$ that form a right coprime factorizations over $\mathbf{R}_{\mathrm{ps}}(z)$ of proper rational matrices $\mathbf{T}_{i}(z)$ given by $(29)$, ie

$$
\mathbf{T}_{i}(z)=\mathbf{N}_{i}(z) \mathbf{D}_{i}^{-1}(z)
$$

Step 3: Check conditions (a), (b) and (c) of the Theorem 1. If all conditions of the Theorem are satisfied go to step 4. If conditions of the Theorem are not satisfied, go to step 5.

Step 4: Set

$$
\mathbf{F}(z)=\left[\mathbf{T}_{1}^{-1}(z)-\mathbf{M}_{1}^{-1}(z)\right]
$$

Step 5: Our problem has no solution.

\section{Conclusions}

In this paper, the problem of simultaneous exact model matching by dynamic output feedback for square and invertible linear time-invariant systems have been studied and completely solved. The problem is studied and solved using coprime factorizations of open-loop transfer function matrices, over the ring of proper and stable rational functions. The main results obtained for linear discrete-time systems hold also for linear continuous-time systems. As we far as we know there are no published results regarding the solvability of simultaneous exact model matching with stability by dynamic output feedback.

The motivation for the study of simultaneous exact model matching problem with stability by dynamic output feedback comes from feedback control of nonlinear systems. In particular, the nonlinear system is represented by linear systems at different operating points [30] and the simultaneous control design consists in finding a single linear feedback controller which guarantees the desired performance for all closed-loop systems. The above clearly demonstrate the contribution of this paper with respect to existing results.

\section{REFERENCES}

[1] V. Blondel, G. Campion and M. Gevers, "Sufficient condition for simultaneous stabilization", EEE Transactions on Automatic Control, vol. 38, no.8, pp. 1264-1266, 1993.

[2] J. Bridwell, D. Castanon and M. Athans, "On reliable control system design with and without feedback reconfiguration", Proceedings of IEEE Conference on Decision and Control, pp. 419-426, 1979.

[3] R. Saeks and J. Murrey, "Fractional reprecentations, algebraic geometry and the simultaneous stabilization problem", IEEE Transactions on Automatic Control, vol. 27 , no.6, pp. 895-903, 1982.

[4] M. Vidyasagar and N. Visvanadham, "Algebraic design techniques for reliable stabilization", IEEE Transactions on Automatic Control, vol. 27, no.5, pp. 1085-1095, 1982.

[5] B. Ghosh, "Trancedental and interpolation methods simultaneous stabilization and simultaneous pole placement problems", 
SIAM Joournal of Control and Optimization, vol. 24, no.6, pp. 1091-1099, 1986.

[6] V. Blondel, Simultaneous stabilization of linear systems, Springer Verlag, Berlin, 1994.

[7] H. Kwakernaak, "A condition for robust stability", Systems and Control Letters, vol. 2, no.2, pp. 1-5, 1982.

[8] A. Saberi, "Simultaneous stabilization with almost disturbance decoupling of uniform rank systems", Automatica, vol. 23, no.5, pp. 653-656, 1987.

[9] E. Emre, "Simultaneous stabilization with fixed closed-loop characteristic polynomial", IEEE Transactions on Automatic Control, vol. 28, no.1, pp. 103-104, 1983.

[10] B. Barmish and K. Wei, "Simultaneous stabilizability of single input single output systems", Proceedings of International Symposium Mathem. Theory of Networks and Systems, Stokholm, 1985.

[11] K. Wei and B. Barmish, "An iterative design procedure for simultaneous stabilization of mimo systems", Automatica, vol. 24, no.5, pp. 643- 652, 1986.

[12] B. T. Polyak and P. S. Shcherbakov, "Hard problems linear control theory :possible approaches to solution", Automation and Remote Control, vol. 66, no.5, pp. 681-718, 2005.

[13] K. Ichikawa, Control system design based on exact model matching techniques, Springer Verlag, Berlin, 1985.

[14] W. Wolovich, "The use of state feedback for exact model matching", SIAM Joournal of Control and Optimization, vol. 10, no.3, pp. 512-523, 1972.

[15] F. S. Wang and C. Desoer, "The exact model matching of linear multivariable systems", IEEE Transactions on Automatatic Control, vol. 17, no.3, pp. 347-349, 1972.

[16] B. Moore and L. Silverman, "Exact model matching by state feedback and dynamic compensation", IEEE Transactions on Automatatic, vol. 17, no.4, pp. 346-354, 1972.

[17] M. Hautus and M. Heymann, "Linear feedback: An algebraic approach", SIAM Journal of Contol and Optimization, vol. 16, no.1, pp. 83- 105, 1978 .

[18] V. Kucera, "Exact model matching polynomial equation approach", International Journal of Sysems and Science, vol. 12, no.12, pp. 1477-1484, 1981.

[19] T. Kaczorek, "Polynomial equation approach to exact model matching problem multivariable linear systems", International Journal of Control, vol. 36, no.3, pp. 531-539.1982.

[20] G. Kimura, T. Matsumoto and S. Takahashi, "A direct Method for exact model matching", Systems and Control Letters, vol. 2, no.1, pp. 531-539, 1982.
[21] A. Morse, "Structure and design of linear model following systems", EEE Transactions on Automatatic Control, vol. 18, no.4, pp. 346-354, 1972.

[22] B. D. O. Anderson and R. Scott, "Parametric solution of the stable exact model matching problem", IEEE Transactions on Automatic Control, vol. 22, no.1, pp. 137-138, 1977.

[23] L. Pernebo, "An algebraic theory for the design of controllers for linear multivariable systems", part I, II., IEEE Transactions on Automatic Control, vol. 26, no.1, pp. 171-194, 1982.

[24] P. J. Antsaklis, "On finite and infinite zeros the model matching problem", Proceedings of 25th IEEE Conference on Decision and Control, pp. 1295-1299, Athens Greece, 1985.

[25] Z. Gao and P. J. Antsaklis, "On stable solutions of the one-and two-sided model matching roblems", IEEE Transactions on Automatic Control, vol. 34, no.9, pp. 978-982.1989.

[26] A. Vardulakis and N. Karcanias, "On the stable exact model matching problem", Systems and Control Letters, vol. 5, no.4, pp. 237-242, .1985.

[27] V. Kucera and E. C. Toledo, "A Review of stable exact model matching by state feedback", 22nd Mediterranean Conferernce on Control and Automation, pp. 85-90, Palermo, Italy, 2014.

[28] C. N. Nett, C. A. Jacobson and N. J. Ballas, "A conection between state-space and doubly coprime fractional representations", IEEE Transactions on Automatic Control, vol. 29, no.9, pp. 831-832, 1984 .

[29] M. Vidyasagar, Control system Synthesis a factorization approach, Morgan \& Claypool, 2011, second edition, 1984.

[30] F. M. Al-Sunni and F. L. Lewis, "Simultaneous output-feedback stabilization for continuous systems", Kybernetika, vol. 34, no.2, pp. 199-202, 1998 .

Received 28 Februay 2016

Konstadinos H. Kiritsis was born in Karditsa, Greece, in 1964. He received the Diploma in mechanical engineering from Czech Technical University of Prague, Prague, Czech Republic, in 1988, and $\mathrm{PhD}$ degree in control theory from School of Electrical and Computer Engineering, National Technical University of Athens, Athens, Greece, in 1998. From 1996 to 2013 he held teaching positions at the Technological Institute of Athens, Technological Institute of Chalkida and Hellenic Air Force Academy. Since April 2014 he is a Lecturer at the Department of Aeronautical Sciences, Hellenic Air Force Academy. His research interests lie in the areas of linear systems and control theory. 